\title{
Escala de Manejo do Dinheiro na Conjugalidade (EMDC): Construção e Evidências de Validade
}

\author{
Lidia Käfer Scbünke ${ }^{1}$ \\ Rebeca Veras de Andrade Vieira ${ }^{1}$ \\ Clarisse Pereira Mosmann ${ }^{1}$ \\ ${ }^{1}$ Universidade do Vale do Rio dos Sinos, São Leopoldo, Rio Grande do Sul, Brasil
}

\begin{abstract}
Resumo
O dinheiro pode ser um fator positivo e/ou negativo na conjugalidade, e a maneira como o casal gerencia os recursos influencia em diferentes aspectos da relação. Este estudo visa apresentar a construção e as propriedades psicométricas da Escala de Manejo do Dinheiro na Conjugalidade (EMDC), elaborada para avaliar os estilos de manejo de dinheiro adotado por indivíduos em relacionamentos estáveis de coabitação: Manejo Independente ou Compartilhado. Participaram 280 homens e mulheres, brasileiros, em relacionamentos estáveis coabitando há, no mínimo, seis meses, sendo $76,4 \%(N=214)$ residentes no estado do Rio Grande do Sul. A partir das análises fatoriais confirmatórias, a escala resultou em 18 itens agrupados em quatro dimensões: Coesão financeira, Intimidade financeira, Infidelidade financeira e Partilha de bens. As análises permitiram identificar estrutura fatorial adequada e evidências de validade. A escala possui potencial para ser utilizada em pesquisa e na prática clínica no âmbito da conjugalidade.
\end{abstract}

Palavras-chave: relações conjugais, finanças, construção do teste, análise fatorial

Conjugality Money Managment Scale (EMDC): Construction and Validity Evidence

\begin{abstract}
The approach couples take to managing financial resources influences different aspects of the marital relationship, and this may correspond to a positive and/or negative factor in conjugality. This study aims to present the construction process and psychometric properties of the Conjugality Money Management Scale (CMMS), designed to evaluate the two possible approaches to money management that can be adopted by individuals in marital cohabitation relationships: Independent or Shared Management approaches. The sample size was 280 participants, including Brazilian men and women, 76.4\% $(\mathrm{N}=214)$ living in the state of Rio Grande do Sul. The confirmatory factorial analyzes resulted in a scale of 18 items, classified into four dimensions: Financial Cohesion, Financial Intimacy, Financial Infidelity, and Asset Sharing. The analyses indicated adequate factorial structure and validity evidence. The scale presents the potential of applicability in research and clinical practice in the context of marital relationships.

Keywords: marital relations; money; test construction; factor analyses
\end{abstract}

\section{Escala de Gestión del Dinero en la Conyugalidad (EMDC): Construcción y Evidencias de Validez}

\begin{abstract}
Resumen
El dinero puede ser un factor positivo y / o negativo en la conyugalidad, y la manera en que una pareja administra sus recursos influye en distintos aspectos de la relación. En este estudio se buscó presentar la construcción y las propiedades psicométricas de la Escala de Gestión del Dinero en la Conyugalidad (EMDC), diseñadas para evaluar los posibles estilos de administración del dinero adoptados por individuos en relaciones estables de convivencia: Gestión Independiente o Compartida. Participaron de la muestra 280 hombres y mujeres de Brasil, en relaciones estables con un mínimo de seis meses de convivencia, con 76.4\% $(\mathrm{N}=214)$ con residencia en la provincia de Rio Grande do Sul. Los 18 ítems resultantes de los análisis factoriales confirmatorios fueron agrupados en cuatro dimensiones: cohesión financiera, intimidad financiera, infidelidad financiera y distribución de activos. Los análisis permitieron identificar una estructura factorial adecuada y evidencias de validez. La escala presenta potencial de aplicabilidad para ser utilizada en investigaciones y prácticas clínicas en el ámbito de las relaciones matrimoniales. Palabras clave: relaciones conyugales; finanzas; construcción de test; análisis factorial
\end{abstract}

\section{Introdução}

Em uma relação conjugal, há a união de duas individualidades, onde cada casal cria sua forma de ser, coexistindo diferentes maneiras de ver o mundo, histórias e projetos de vida (Fères-Carneiro, 1998). A forma como a conjugalidade irá se constituir conecta-se às questões de ordem pessoal, interpessoal, cultural, social e transgeracional (Diniz, 2011). O processo de desenvolvimento humano e os relacionamentos são complexos, o que aponta para a importância de compreender os fenômenos que envolvem esse contexto.

Um dos aspectos que perpassa diferentes esferas da vida e está presente no cotidiano é o dinheiro, que vem sendo explorado na literatura a partir de distintas perspectivas teóricas e implicações no contexto 
individual e social (Barros, Borges, \& Estramiana, 2017). Nas relações conjugais, ele pode atuar como um fator positivo e/ou negativo, sendo a forma de gerenciamento um dos aspectos a serem considerados nessa esfera (Garbin, Mara, Cenci, \& Luz, 2015).

A forma como um casal estabelece o manejo dos recursos financeiros tem relação com os níveis de satisfação conjugal, comunicação, confiança e respeito (Harth \& Falcke, 2017), bem como sentimentos positivos ou negativos em relação ao cônjuge e à união (Papp, Cummings, \& Goeke-Morey, 2009). As formas como os casais gerenciam os recursos financeiros vem sendo estudadas, considerando seu impacto em diferentes aspectos da vida conjugal, como ajustamento, qualidade e conflitos (Cenci, Bona, Crestani, \& Habigzang, 2017).

Em um sistema de gestão independente, cada um dos membros do casal irá gerenciar e controlar individualmente sua renda e suas obrigações individuais com as despesas, e os cônjuges não têm acesso ao dinheiro um do outro. Já em um sistema de gestão compartilhada, tanto o acesso aos recursos quanto as decisões financeiras são compartilhadas por ambos os cônjuges (Pahl, 1989).

A literatura aponta relações entre os estilos de manejo e a qualidade conjugal (Harth \& Falcke, 2017; Razera, Cenci, \& Falcke, 2015; Papp et al., 2009), sendo que estilos de manejo em conjunto apontam para maior qualidade conjugal (Cenci et al., 2017). Sistemas de gerenciamento individuais aparecem associados com a diminuição de sentimentos de intimidade, satisfação sexual e satisfação em relação à resolução de conflitos por parte das mulheres (Addo \& Sassler, 2010). Ainda, nos casais onde o manejo do dinheiro é compartilhado, é favorecida a afirmação da união conjugal, da intimidade e da afetividade (Archuleta, 2013).

Apesar das relações apontadas na literatura, percebe-se uma carência instrumental para mensurar o fenômeno, uma vez que diferentes pesquisas nas últimas décadas se utilizam de entrevistas e questionários sem contar com um instrumento específico para avaliação. Na década de 1980, a partir de dados da Iniciativa de Mudança Social e Econômica do Reino Unido, foi analisada a relação entre dinheiro, poder e desigualdade no casamento constatando questões sobre o gerenciamento das finanças. Entretanto, os dados foram levantados por meio de entrevistas individuais e questionários (Vogler \& Pahl, 1993).

$\mathrm{Na}$ década de 1990, uma análise do perfil de gastos familiares de 3.676 casais britânicos envolveu sete grupos focais com 59 indivíduos e a condução de entrevistas presenciais com 40 casais residentes na Inglaterra. Tal método visou compreender a forma que indivíduos e casais manejam suas finanças (Pahl, 2000). Burgoyne, Clarke, Reibstein e Edmunds (2006), ao estudarem aspectos financeiros de 42 casais heterossexuais no sul da Inglaterra, levantaram dados por meio de entrevistas semiestruturadas. Percebe-se a valorização da temática na Europa, em destaque no Reino Unido, ainda que o acesso seja qualitativo.

Outras pesquisas conduzidas sobre manejo do dinheiro na conjugalidade mensuram o fenômeno por meio de uma única questão, a partir da qual os indivíduos respondem de que forma o dinheiro é gerenciado, se apenas por um ou por ambos (Cardoso \& Bucher-Maluschke, 2017; Cenci \& Habigzang, 2019; Cenci, Pauli, \& Folle, 2018; Cenci et al., 2017; Harth, Mosmann, \& Falcke, 2016; Yodanis \& Lauer, 2007). No Brasil, são recentes os estudos que abarcam a temática dos estilos de manejo do dinheiro.

Archuleta (2008) desenvolveu um instrumento com propriedades psicométricas para verificar o envolvimento de cada cônjuge no gerenciamento das finanças dentro de um contexto rural entre casais residentes em estados do oeste dos Estados Unidos. A Financial Managment Roles (FMR) foi composta por 19 itens avaliados em uma escala Likert de 1 a 7 , que mensurava a responsabilidade pessoal, conjunta ou do cônjuge em relação ao gerenciamento das finanças na propriedade rural e obteve alfa de Cronbach de 0,930. Contudo, a FMR é voltada para a conjugalidade e a administração financeira especificamente no meio rural. Os respondentes devem indicar sua responsabilidade na execução de tarefas, como bookkeeper, employee wages, farm related insurance e livestock, purchase/sales (contabilidade, salário dos funcionários, seguro da fazenda e compra/venda de gado, em tradução livre), não sendo passível de replicação em diferentes cenários.

O estilo de manejo do dinheiro adotado por um casal abarca questões complexas e envolve diferentes aspectos de ordem individual, transgeracional, conjugal e social (Barros et al., 2017; Pahl, 2005). A compreensão do fenômeno requer o entendimento de como o casal estabelece e divide as responsabilidades em relação às finanças, qual o nível de intimidade financeira que compartilham, se ocorre a infidelidade financeira e como se dá a partilha dos bens (Cenci et al., 2017; Hart et al., 2016; Archuleta, 2013; Pahl, 2005).

A temática vem sendo estudada de forma estruturada há pelo menos três décadas e o cenário apresentado denota a carência instrumental para 
mensurar o fenômeno. Medidas de avaliação servem como importantes ferramentas para pesquisadores e profissionais, pois possibilitam maior precisão, previsão e possibilidades de estimativas. A possibilidade de replicação de estudos é valorizada na Psicologia (Reppold et al., 2015). Assim, o objetivo deste estudo foi construir e verificar evidências de validade de uma escala para identificar os estilos de manejo do dinheiro na conjugalidade para a população brasileira, a EMDC (Escala de Manejo do Dinheiro na Conjugalidade).

\section{Método}

\section{Elaboração do Instrumento}

A partir de uma revisão teórica da literatura sobre o manejo financeiro e o fenômeno do dinheiro na conjugalidade, procedeu-se com a elaboração da Escala de Manejo do Dinheiro na Conjugalidade (EMDC). O processo de construção dos itens e definição das quatro dimensões emergiu da literatura especializada na área (Archuleta, 2013; Barros et al., 2017; Cardoso \& Bucher-Maluschke, 2017; Cenci \& Habigzang, 2019; Cenci et al., 2018; Cenci et al., 2017; Garbin et al., 2015; Hart et al., 2016; Pahl, 2005; 1989; Yodanis e Lauer, 2007) e discussão com juiz especialista em conjugalidade: Coesão financeira tange à responsabilidades compartilhadas em relação às finanças, considerando as despesas, a tomada de decisão e as ações envolvendo o uso do dinheiro; Intimidade financeira abrange a partilha de informações, valores, planejamento financeiro e opinião em relação ao dinheiro; Infidelidade financeira abarca atitudes de esconder do(a) parceiro(a) ações e informações referentes a gastos, ganhos e/ou investimentos financeiros; Partilha de bens, por fim, refere-se a como o casal percebe e gerencia a propriedade dos recursos financeiros.

Foram utilizados os procedimentos para análise da qualidade dos itens desenvolvidos de acordo com critérios de pertinência prática (se o item pertence ao fenômeno estudado) e clareza de linguagem (se a forma como o item está escrito é clara, compreensível e apropriada) (Pasquali, 2011), os quais foram submetidos à análise de cinco juízes especialistas em terapia de casal. A avaliação foi feita por meio de uma escala Likert de 5 pontos, sendo que uma pontuação 0 representou nenhuma pertinência e linguagem inapropriada e 4 representou um item pertinente expresso por meio de uma linguagem precisa. Os itens receberam concordância em relação à pertinência prática $(\mathrm{CVC}=0,990)$. No que tange à clareza de linguagem $(\mathrm{CVC}=0,830)$, após análise dos juízes, foram feitas as adequações semânticas.

A escala resultou em 19 itens em uma escala Likert de 7 pontos, de Não aplicável a Muito aplicável, contendo afirmações como "Eu e meu parceiro partilhamos a responsabilidade pelas despesas domésticas" e "Eu e meu parceiro planejamos nossos gastos em conjunto". Os itens resultantes foram submetidos à análise de compreensão por três pessoas que estavam em um relacionamento estável no momento. As idades variaram entre 29 e 62 anos $(M=43 ; D P=17,06)$, sendo duas pessoas com escolaridade de ensino superior e uma pessoa com nível médio. Os 19 itens foram bem compreendidos e compuseram então a versão preliminar da escala a ser testada empiricamente.

Para identificar o estilo de manejo do dinheiro, foi utilizado o ponto de corte de 76. Escore inferior a 76 pontos caracterizaria estilo de manejo independente $\mathrm{e}$ escore superior a 76 pontos denotaria estilo de manejo do dinheiro compartilhado.

\section{Participantes}

Participaram do estudo 280 homens e mulheres, brasileiros, que estavam em um relacionamento e coabitando há, no mínimo, seis meses, sendo 76,4\% (N $=214$ ) residentes no estado do Rio Grande do Sul. O tempo total de relacionamento variou de 12 meses a 51,08 anos $(M=14,22$ anos; $D P=11,43$ anos $)$ e o de coabitação variou de 6 meses a 47,08 anos $(M=10,84$ anos; $D P=11,04$ anos). Dentre os participantes, $94,6 \%$ $(N=265)$ declararam ter a ocupação profissional como fonte de renda. A Tabela 1 apresenta as características sociodemográficas da amostra.

Cabe ressaltar que essa amostra é composta majoritariamente por indivíduos residentes na Região Sul, que exercem atividade remunerada e cursaram graduação. A amostra possui nível socioeconômico superior à média nacional, uma vez que, em 2015, 13,5\% da população maior de 25 anos de idade possuía ensino superior completo e o rendimento médio brasileiro foi de 2,35 salários mínimos mensais (IBGE, 2015). É importante considerar o perfil específico dessa amostra.

\section{Instrumentos}

Além da versão preliminar da EMDC, foi utilizado um instrumento para avaliar o ajustamento conjugal, um para comunicação conjugal, e os participantes responderam a um questionário sociodemográfico. Este foi utilizado para fins de caraterização da amostra e continha questões, como idade, sexo, situação conjugal, 
Tabela 1.

Caracterização Sociodemográfica dos Participantes

\begin{tabular}{lcc}
\hline Variáveis & Média & $D P$ \\
\hline Idade & 37,82 & 11,524 \\
Renda Mensal em salários mínimos* & & \\
$\quad$ Individual & 5,33 & 5,19 \\
$\quad$ Familiar & 10,28 & 9,26 \\
\hline & $N$ & $\%$ \\
\hline Gênero & 177 & 63,2 \\
$\quad$ Feminino & 103 & 36,1 \\
$\quad$ Masculino & & \\
Situação Conjugal & 189 & 67,5 \\
$\quad$ Casamento civil / religioso ou ambos & 91 & 32,5 \\
$\quad$ Morando juntos / União estável & 237 & 84,6 \\
Indivíduos em primeira união conjugal & 143 & 51,91 \\
Indivíduos com filhos & & \\
Escolaridade & 2 & 0,7 \\
$\quad$ Ensino Fundamental & 26 & 9,3 \\
Ensino Médio & 19 & 6,8 \\
Ensino Técnico & 124 & 44,3 \\
Ensino Superior & 109 & 38,9 \\
Pós-Graduação & & \\
\hline
\end{tabular}

*Salário Mínimo 2019: R\$998,00 (Brasil, 2019).

filhos, situação laboral, escolaridade, renda, tempo de relacionamento e filhos.

A R-DAS (Hollist et al., 2012) é uma versão reduzida da DAS - Dyadic Adjustment Scale, questionário de autorrelato dirigido à avaliação da satisfação com a relação, a sua coesão e expressão de afeto (Rosado \& Wagner, 2015). A R-DAS contêm 14 itens que avaliam a percepção de ajustamento diádico do indivíduo e, neste estudo, foi utilizada a dimensão Coesão para verificar evidências de validade concorrente com a Coesão financeira. Apresenta bons índices de consistência interna, com alfa de Cronbach de 0,800 para coesão. Esses resultados indicam qualidade psicométrica. Destaca-se que esse é um dos instrumentos mais utilizados para avaliação da satisfação conjugal, inclusive no contexto brasileiro (Pereira-Silva, 2015; Peruchi, Donelli, \& Marin, 2016). Neste estudo, verificou-se alfa de Cronbach de 0,872 para a escala total e 0,824 para a dimensão Coesão.

Para avaliar a comunicação conjugal, utilizou-se a escala Dutch Marital Satisfaction e Communication
Questionnaire - DMSCQ (Troost, Vermulst, Gerris, \& Matthjis, 2005), a qual objetiva mensurar satisfação e comunicação conjugal por meio de três dimensões: Satisfação conjugal, Comunicação negativa e Comunicação aberta. Neste estudo, foram utilizadas as duas dimensões referentes à comunicação, as quais consistem em 15 itens pontuados em escala Likert de 7 pontos, variando de Não aplicável a Muito aplicável.

Luz e Mosmann (2018) procederam com a tradução da escala para a língua portuguesa, por meio do processo de back translation realizado por especialistas, para garantir a qualidade semântica, equivalência cultural entre as fontes e equivalência normativa com a pesquisa. A dimensão Comunicação negativa é composta por nove itens que mensuram em que grau o indivíduo relata a experiência de comunicação na relação conjugal como negativa. Já a dimensão Comunicação Aberta afere o nível em que o respondente relata troca de experiências pessoais com o parceiro conjugal, por meio de seis itens. No processo de adaptação,

Psico-USF, Bragança Paulista, v. 26, n. 2, p. 305-318, abr./jun. 2021 
o instrumento apresentou com alfa de Cronbach para comunicação negativa de 0,740 e, para a comunicação aberta, de 0,700 .

O DMSCQ foi utilizado para verificar evidências de validade concorrente da dimensão de intimidade financeira da escala, uma vez que a comunicação exerce influência direta na intimidade conjugal (Bereza, Martins, Moresco, \& Zanoni, 2005). O instrumento foi aplicado por se tratar de uma escala adaptada para a população brasileira. Neste estudo, a dimensão Comunicação Aberta apresentou índices consistência interna com alfa de Cronbach 0,834.

\section{Procedimentos Éticos e de Coleta de Dados}

A coleta de dados foi realizada pela internet, por meio de questionário on-line hospedado na plataforma Google Forms. A divulgação se deu por meio de um flyer compartilhado em redes sociais convidando as pessoas a participarem, bem como enviado por e-mail e aplicativos de mensagens a grupos de diferentes locais do Brasil. A divulgação da pesquisa foi incentivada entre os participantes. Ao acessar o formulário, eles tiveram acesso ao Termo de Consentimento Livre Esclarecido, contendo os termos e objetivos da pesquisa, bem como os procedimentos éticos adotados. Para acessar o questionário, era necessário que os sujeitos consentissem sua participação de forma livre e voluntária. A pesquisa foi aprovada pelo comitê de ética da Universidade do Vale do Rio dos Sinos (CAAE 03878618.2.0000.5344), atendendo às normas do Conselho Nacional de Saúde para pesquisa envolvendo seres humanos (Brasil, 2016; Brasil 2012). O questionário permaneceu ativo durante os meses de janeiro e fevereiro do ano de 2019.

\section{Análise dos Dados}

Antes de conduzir a análise fatorial da escala, foram verificados os critérios de normalidade da amostra no software Statistical Package for Social Sciences 22.0 (IBM SPSS) por meio do teste de Shapiro-Wilk, recomendado para tamanhos amostrais maiores do que 100 (Torman, Coster, \& Riboldi, 2012). Verificou-se que a distribuição dos dados é assimétrica, requerendo então o cálculo do escore $Z$ referente aos resultados da escala.

Adicionalmente, foram conduzidas análises de associação entre os itens da escala visando identificar a existência de correlações fortes $(>0,800)$. Foram verificadas duas correlações fortes e estatisticamente significativas entre itens: "Meu/minha parceiro(a) tem acesso às informações referentes aos meus gastos" e "Eu tenho acesso às informações referentes aos gatos do meu/minha parceiro(a)" ( $r=0,825)$; "Eu e meu/ minha parceiro(a) mantemos uma conta conjunta para gastos específicos (despesas domésticas, lazer, viagens, gastos com filhos etc.)" e "Eu e meu/minha parceiro(a) mantemos uma conta conjunta para todos os nossos gastos" $(r=0,842)$. Procedeu-se com análise de conteúdo dos itens e optou-se por mantê-los na escala por avaliarem fenômenos diferentes: o primeiro item diz respeito ao cônjuge, e o segundo item se refere especificamente ao respondente. A existência de reciprocidade dentro de uma relação conjugal pode justificar essa correlação forte, porém, ainda assim, trata-se de questões distintas. Já a manutenção das contas conjuntas pode ocorrer para objetivos diferentes dentro da relação, de forma total ou parcial (Pahl, 1989). Dessa forma, visando contemplar o manejo do dinheiro na conjugalidade de forma ampla, não se justifica a exclusão dos itens.

Posteriormente, foi realizada uma análise fatorial confirmatória (AFC) em Modelagem de Equações Estruturais (SEM) com os 19 itens propostos, considerando as dimensões geradas a partir da revisão de literatura: Coesão Financeira, Intimidade Financeira, Infidelidade Financeira e Partilha de Bens. Sendo a AFC um método rigoroso e restritivo que analisa as relações entre um conjunto de variáveis observadas e variáveis latentes, requer sustentação teórica do modelo proposto e relação entre os fatores (Byrne, 2010). Esse procedimento visa a confirmação de quais variáveis definem os construtos, sendo que na AFC o modelo é derivado da teoria e, então, testado para indicar a consistência dos dados observados (Raykov \& Marcoulides 2000).

Brown (2006) sustenta sua utilização no processo de desenvolvimento de uma escala para testar a adequação do modelo teoricamente proposto e o padrão de interação entre os itens e os fatores. A abordagem confirmatória leva os pesquisadores a otimizar o processo de elaboração de um instrumento e a proporciona maior confiabilidade e validade em relação às abordagens exploratórias e ao índice alfa de Cronbach (Batista-Foguet, Coenders, \& Alonso, 2004; Brown, 2006).

Para fins de estimação, foi utilizado o método de Maximum Likelihood. A AFC foi realizada por meio do software Statistical Package for Social Sciences Analysis of Moment Structures 2.0 (IBM SPSS AMOS). Os índices de ajuste considerados para a adequação do modelo foram a razão do qui-quadrado em relação aos graus de liberdade $\left(x^{2} / g\right)$, o Comparative Fit Index (CFI), o Tucker-Lewis 
Index (TLI) e o Root Mean Square Error of Approximation (RMSEA). Em relação à $x^{2} / g l$, valores entre 1 e 3 são indicadores de bom ajuste do modelo (Kline, 2005). O CFI e o TLI requerem valores superiores a 0,900 e o RMSEA, valor inferior a 0,080 (Byrne, 2010; Hair, Black, Babin, Anderson, \& Tatham, 2009).

Para verificar evidências de validade concorrente, foi utilizada a correlação de Spearman entre os itens da dimensão Coesão Financeira e a R-DAS, procedimento repetido entre a dimensão Intimidade Financeira e a DMSCQ, uma vez que são construtos teoricamente relacionados (Pasquali, 2011). Não foram verificadas na literatura a disponibilidade de instrumentos validados que avaliam construtos correlatos à Infidelidade Financeira e Partilha de Bens a partir de uma perspectiva da conjugalidade, de forma que não foi possível verificar evidências de validade concorrente para essas dimensões.

\section{Resultados}

Foram testados dois modelos: um unidimensional e outro com a estrutura fatorial proposta. $\mathrm{O}$ modelo de quatro fatores foi testado com e sem a possibilidade de variância entre os itens. A Tabela 2 apresenta os índices de ajuste apurados para cada um dos modelos testados.

Conforme os índices $x^{2} / g l$, CFI, TLI e RMSEA, verifica-se que o modelo unidimensional não se ajustou aos dados. Porém, a análise do peso de regressão dos itens $(<0,500)$ indicou a exclusão dos itens que, no modelo de quatro fatores, diziam respeito à dimensão Infidelidade Financeira - o que poderia favorecer o ajuste. A utilização de SEM requer que os modelos sejam compreendidos à luz da teoria que os fundamenta (Hair et al., 2009), de forma que se entende a infidelidade financeira como um fenômeno importante (Cenci \& Habigzang, 2019) ao considerar as práticas de manejo do dinheiro na conjugalidade, o que inviabiliza o procedimento. Não havendo sustentação teórica para a exclusão das práticas de infidelidade financeira ao mensurar o manejo do dinheiro na conjugalidade, o modelo unidimensional não se adequou aos dados.

O modelo de quatro fatores relacionados apresentou o índice de ajuste CFI adequado, e os índices $x^{2} /$ $d f$, TLI e RMSEA situaram-se próximos ao limite dos pontos de corte. Em análise do peso de regressão dos itens em relação às dimensões, observou-se que o item 13 ("Fazemos investimento financeiros juntos"), apesar de possuir significância estatística $(\phi<0,001)$, apresentou coeficiente padronizado $(0,491)$ inferior ao limite mínimo de 0,500 (Hair et al., 2009). Considerando a hipótese de o item estar interferindo na adequação do modelo, foi conduzida nova análise após a exclusão deste, resultando em valores de ajustamento satisfatórios no que tange aos índices $x^{2} / d f(2,671)$, CFI $(0,933)$ TLI $(0,915)$ e RMSEA $(0,077)$ - com intervalo de confiança de $90 \%(0,067-0,088)$. Com a exclusão do item, o ponto de corte da escala passou a ser 72 pontos. Um escore inferior a 72 pontos representa Manejo Independente, e um escore superior a 72 pontos refere-se à adoção de um sistema de Manejo Compartilhado.

A Tabela 3 mostra os coeficientes padronizados dos itens e as estimativas dos parâmetros do modelo. Os resultados dos coeficientes apresentaram valores satisfatórios acima de 0,500, indicando associação dos itens aos fatores. Tais resultados corroboram a designação teórica dos itens às respectivas dimensões. A Figura 1 mostra o modelo final da Escala de Manejo do Dinheiro na Conjugalidade.

A análise de validade concorrente da dimensão Coesão Financeira apresentou correlação positiva com a dimensão Coesão da R-DAS ( $r=0,468)$, com valor de $p<0,001$. A correlação é moderada e estatisticamente significativa.

Já a dimensão Intimidade Financeira da EMDC correlacionou, no sentido esperado, com as duas dimensões da DMSCQ: Comunicação Negativa $(r=-0,278)$ e Comunicação Aberta $(r=0,354)$ com valor de $p<$ 0,001. As associações são estatisticamente significativas, porém são correlações fracas. É importante considerar que, apesar de ambas avaliarem a comunicação

Tabela 2.

Comparação entre os Índices de Ajuste e Resíduo dos Modelos Testados

\begin{tabular}{llllll}
\hline Modelo & $X^{2} / d f$ & CFI & TLI & RMSEA & IC 90\% \\
\hline Unidimensional & 9,511 & 0,594 & 0,544 & 0,175 & $0,166-0,183$ \\
Quatro Fatores & 4,803 & 0,826 & 0,796 & 0,117 & $0,108-0,125$ \\
Quatro Fatores com covariância & 3,082 & 0,910 & 0,888 & 0,086 & $0,077-0,096$ \\
\hline
\end{tabular}


Tabela 3.

Estatísticas Descritivas dos Itens e Estimativas dos Parâmetros do Modelo

\begin{tabular}{|c|c|c|c|c|}
\hline Item & Dimensão & $\begin{array}{l}\text { Coeficiente } \\
\text { Padronizado }\end{array}$ & $\begin{array}{l}\text { Estimativa } \\
\text { de erro }\end{array}$ & Z \\
\hline $\begin{array}{l}1 \text { - Eu e meu/minha parceiro(a) partilhamos a } \\
\text { responsabilidade pelas despesas domésticas. }\end{array}$ & \multirow{3}{*}{$\begin{array}{l}\text { Coesão } \\
\text { Financeira }\end{array}$} & 0,811 & 0,064 & $12,647 *$ \\
\hline $\begin{array}{l}2 \text { - Eu e meu/minha parceiro(a) dividimos a responsabilidade } \\
\text { pela tomada de decisões que envolvem dinheiro. }\end{array}$ & & 0,886 & 0,053 & $16,584^{*}$ \\
\hline $\begin{array}{l}3 \text { - Eu e meu/minha parceiro(a) conversamos sobre o uso do } \\
\text { dinheiro. }\end{array}$ & & 1,000 & & \\
\hline $\begin{array}{l}4 \text { - Eu tenho acesso às informações referentes aos gastos } \\
\text { do(a) meu/minha parceiro(a). }\end{array}$ & \multirow{8}{*}{$\begin{array}{l}\text { Intimidade } \\
\text { Financeira }\end{array}$} & 0,782 & 0,059 & $13,340^{*}$ \\
\hline $\begin{array}{l}5 \text { - Meu/minha parceiro(a) tem acesso às informações } \\
\text { referentes aos meus gastos. }\end{array}$ & & 0,783 & 0,058 & $13,560^{*}$ \\
\hline 6 - Sei o quanto de dinheiro meu/minha parceiro(a) ganha. & & 0,726 & 0,056 & $12,944 *$ \\
\hline $\begin{array}{l}7 \text { - Meu/minha parceiro(a) sabe o quanto de dinheiro eu } \\
\text { ganho. }\end{array}$ & & 0,545 & 0,049 & $11,223 *$ \\
\hline $\begin{array}{l}10 \text { - Eu e meu/minha parceiro(a) podemos usar o dinheiro } \\
\text { um do outro. }\end{array}$ & & 0,881 & 0,065 & $13,559 *$ \\
\hline $\begin{array}{l}11 \text { - Eu e meu/minha parceiro(a) anotamos em conjunto } \\
\text { nossos gastos. }\end{array}$ & & 0,814 & 0,070 & $11,599 *$ \\
\hline $\begin{array}{l}12 \text { - Eu e meu/minha parceiro(a) planejamos nossos gastos } \\
\text { em conjunto. }\end{array}$ & & 1,000 & & \\
\hline $\begin{array}{l}14 \text { - Eu levo em consideração a opinião do(a) meu/minha } \\
\text { parceiro(a) em relação ao uso do dinheiro. }\end{array}$ & & 0,794 & 0,050 & $15,891 *$ \\
\hline 15 - Eu já escondi gastos do meu/minha parceiro(a). & \multirow{4}{*}{$\begin{array}{l}\text { Infidelidade } \\
\text { Financeira }\end{array}$} & 0,701 & 0,104 & $6,742^{*}$ \\
\hline $\begin{array}{l}16 \text { - Eu desconfio que meu/minha parceiro(a) já escondeu } \\
\text { gastos de mim. }\end{array}$ & & 1,000 & & \\
\hline $\begin{array}{l}18 \text { - Desconfio que meu/minha parceiro(a) tenha dinheiro } \\
\text { guardado sem que eu saiba. }\end{array}$ & & 0,687 & 0,102 & $6,749^{*}$ \\
\hline $\begin{array}{l}19 \text { - Eu tenho dinheiro guardado sem que meu/minha } \\
\text { parceiro(a) saiba. }\end{array}$ & & 0,593 & 0,096 & $6,201 *$ \\
\hline $\begin{array}{l}8 \text { - Eu e meu/minha parceiro(a) mantemos uma conta } \\
\text { conjunta para gastos específicos (despesas domésticas, lazer, } \\
\text { viagens, gastos com filhos etc.). }\end{array}$ & \multirow{3}{*}{$\begin{array}{l}\text { Partilha de } \\
\text { Bens }\end{array}$} & 0,964 & 0,053 & $18,687^{*}$ \\
\hline $\begin{array}{l}9 \text { - Eu e meu/minha parceiro(a) mantemos uma conta } \\
\text { conjunta para todos os nossos gastos. }\end{array}$ & & 1 & & \\
\hline $\begin{array}{l}17 \text { - Eu e meu/minha parceiro(a) compartilhamos um cartão } \\
\text { de crédito e/ou débito. }\end{array}$ & & 0,562 & 0,058 & $9,678^{*}$ \\
\hline
\end{tabular}

Nota. $*_{p}<0,001$

na conjugalidade, a DMSCQ avalia como o indivíduo percebe a experiência da comunicação na relação conjugal (Luz e Mosmann, 2018). A EMDC avalia a intimidade dentro do contexto específico do manejo do dinheiro, construto que possui correlação forte com a dimensão Coesão Financeira $(r=0,704)$ e moderada com as dimensões Partilha de Bens $(r=0,540)$ e Infidelidade Financeira $(r=-0,495)$, indicando que fatores correlatos às outras dimensões perpassam os processos de comunicação referentes ao manejo financeiro na 


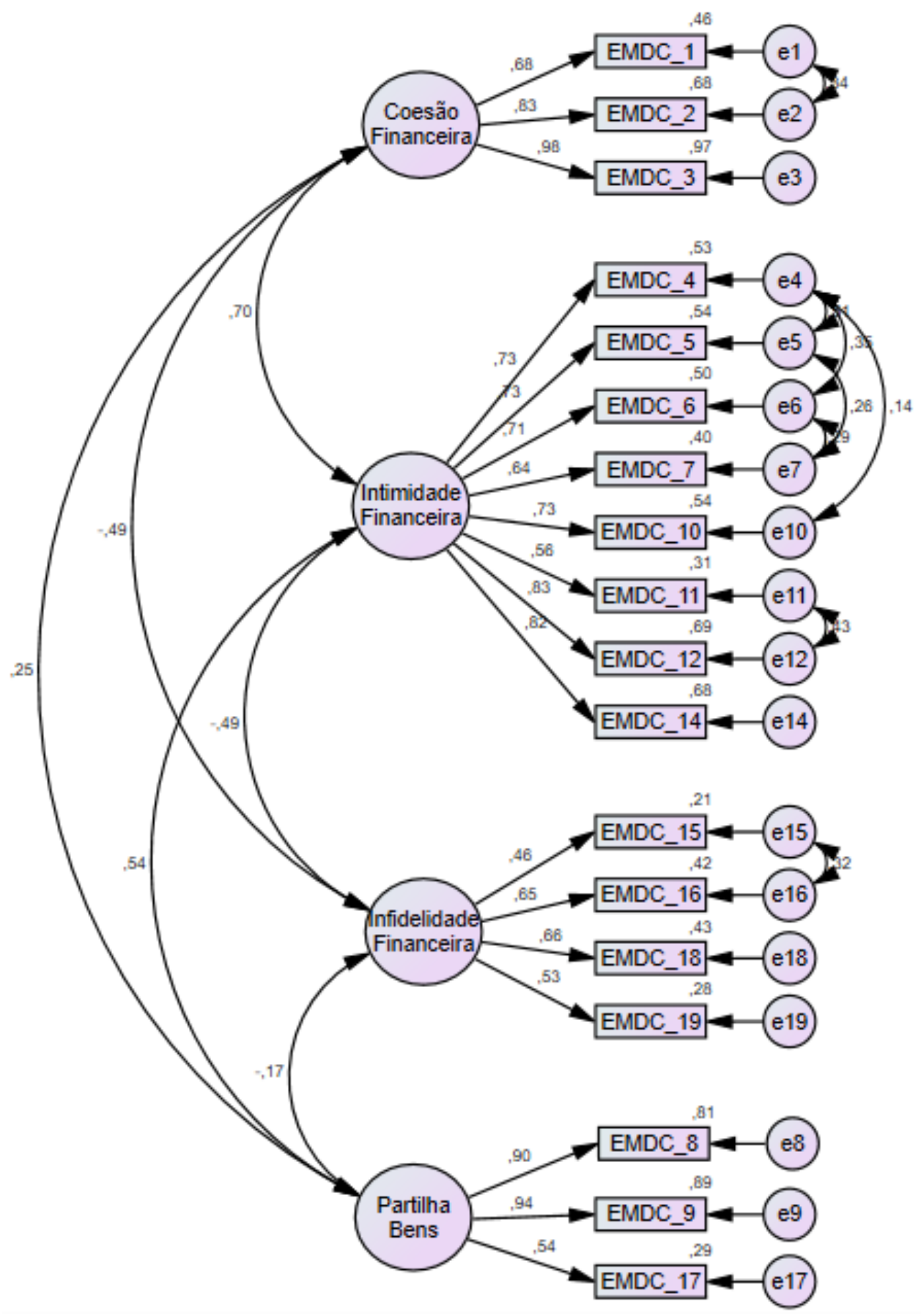

Figura 1. Modelo final EMDC.

relação conjugal. Dessa forma, os resultados apontam para validade concorrente das dimensões avaliadas.

\section{Discussão}

Este estudo objetivou a construção de uma escala de avaliação do manejo do dinheiro na conjugalidade para a população brasileira, considerando os estilos de manejo individual e compartilhado (Pahl, 1989). O processo de construção da EMDC resultou inicialmente em uma escala com 19 itens distribuídos em quatro dimensões: Coesão Financeira, Intimidade Financeira, Infidelidade Financeira e Partilha de Bens. Foram conduzidas análises fatoriais confirmatórias e de correlação, 
que forneceram evidências de validade de construto para estrutura da escala com 18 itens agrupados nas dimensões correspondentes.

A análise fatorial confirmatória do modelo proposto requereu a exclusão do item "Fazemos investimento financeiros juntos", fato que aponta para as características sociodemográficas e práticas culturais da população brasileira. Segundo o Indicador de Reserva Financeira do Brasileiro, a partir de estudo realizado em 12 capitais do país no ano de 2018, apenas 16,2\% dos participantes conseguiram efetuar alguma reserva financeira nesse período. No ano de 2017, esse índice foi de 21\% (SPC Brasil \& CNDL, 2018; SPC Brasil \& CNDL, 2017). Esse cenário é corroborado pelo relatório da Comissão de Valores Mobiliários, autarquia vinculada ao Ministério da Fazenda, o qual aponta que também no ano de 2018 apenas 27\% dos brasileiros possuíam como hábito a realização de investimentos financeiros (CVM, 2018).

Cabe ressaltar ainda que a realização de investimentos financeiros não é prática comum entre a população brasileira, uma vez que, no ano de 2017, apenas 9\% da população possuía capital investido em algum nível (Anbima, 2018). Os relatórios citados apontam que a prática de guardar dinheiro na caderneta de poupança é considerada uma forma de investimento, sendo a mais utilizada pelos brasileiros.

Entende-se que fatores culturais, a conjuntura socioeconômica e a relação entre o poder aquisitivo médio e a demanda de gastos necessários para subsistência das famílias brasileiras são fatores que contribuem para esse cenário onde a prática de investimentos financeiros não é um hábito recorrente ou, em muitos casos, possível: o percentual de famílias brasileiras que possuem algum nível de endividamento atingiu o nível de 60,5\% no mês de fevereiro do corrente ano (CNC, 2019). Em tempo, são compreendidas como investimentos financeiros também a aquisição de imóveis e demais bens a fim de aumentar o patrimônio familiar. O perfil socioeconômico da amostra acessada no presente estudo pode ter contribuído para a prática de investimentos financeiros, o que explicaria o valor tão aproximado ao ponto de corte para a regressão do item. Diante do exposto, justifica-se a exclusão deste.

A maneira como os casais dividem as responsabilidades envolvendo o dinheiro é um dos determinantes do estilo de manejo adotado. Aqui, entende-se que as responsabilidades abrangem não só os compromissos financeiros, mas também a tomada de decisões (Addo \& Sassler, 2010; Cenci et al., 2017). A dimensão Coesão
Financeira (CF) mensura qual o nível de coesão dos casais em relação a essas questões. Olson (2000) postula que a coesão diz respeito ao vínculo emocional existente entre os indivíduos, mas consiste também em como um sistema equilibra a sua separação e união ao mesmo tempo. Dentro desses processos, ressalta-se a tomada de decisões e o estabelecimento de limites.

Em relação às finanças, a forma como um casal partilha as responsabilidades e estabelece os limites denota o quanto ambos compartilham um vínculo frente à administração do dinheiro. Estudo com 1.292 pessoas em relacionamentos estáveis de coabitação na Grã-Bretanha verificou que aqueles que tomavam decisões financeiras de forma individual demonstravam maior insatisfação em relação a sua vida familiar do que aqueles que o faziam de forma conjunta (Vogler, Lyonette, \& Wiggings, 2008). Os níveis de CF apontam como se dá a conexão da díade diante das experiências envolvendo as finanças, e a análise fatorial dos itens demonstrou que tanto a conversa sobre o uso do dinheiro quanto o compartilhamento das responsabilidades e da tomada de decisões contribuem positivamente para o desfecho desse construto.

A intimidade conjugal é um dos aspectos mais valiosos de uma relação, ocupando espaço de grande importância para a conexão de um casal (Prager, 2014), promovendo a continuidade da relação (Crespo, Narciso, Ribeiro, \& Costa, 2006). O nível de compromisso entre duas pessoas, a proximidade cognitiva, física e os afetos positivos irão determinar o nível de intimidade (Moss \& Shwebel, 1993). O compartilhamento de algo íntimo de si e a percepção de que isso é recebido, compreendido e valorizado pelo outro irão definir a intimidade (Levine, 1992). Dentre diferentes acepções teóricas, o que existe em comum é o ato de dividir com o outro algo privado e particular.

A Intimidade Financeira contempla a partilha de informações e recursos financeiros entre os cônjuges, possibilitando proximidade e conexão diante de algo que é considerado privado e particular como o dinheiro (Barros et al., 2017). Na EMDC, os itens de acesso às informações financeiras e compartilhamento de valores apresentaram valores satisfatórios para medir a dimensão.

A infidelidade é um fenômeno complexo nas relações conjugais. Caracterizada pela mentira, omissão ou envolvimento físico/emocional com terceiros pode estar relacionada a diferentes fatores, como desequilíbrio conjugal ou perda de interesse sexual e diminuição da intimidade (Haack \& Falcke, 2013). Esse fenômeno 
ocorre de formas distintas, dentre elas a infidelidade financeira entre os cônjuges: quando um (ou ambos) omitem ou alteram informações referentes a gastos, investimentos ou dívidas (Hart et al., 2016).

A dimensão Infidelidade Financeira apresentou relevância estatística na mensuração do construto Manejo do Dinheiro, pois tem consequências diretas tanto na manutenção quanto nos possíveis desfechos da relação. Pode contribuir para o surgimento de importantes conflitos conjugais (Cenci \& Habigzang, 2019; Cenci et al., 2017; Hart et al., 2016; Sattler, Tavares, \& Silva, 2017). A correlação negativa verificada entre essa dimensão e as demais denota o quanto tais comportamentos impactam negativamente no manejo financeiro na conjugalidade.

Ao dinheiro pode ser atribuído um significado de poder, assumindo características de dominação (Rosa \& Milani, 2014). O estabelecimento e a divisão de poder nas relações são aspectos que interferem na construção, manutenção e dissolução da conjugalidade (Fères-Carneiro \& Diniz Neto, 2010). Diante disso, é necessário compreender como se organiza a partilha dos recursos financeiros (Archuleta, 2013; Pahl, 2005). A análise fatorial da EMDC demonstrou que a manutenção de contas conjuntas, seja para todos os gastos ou gastos específicos, bem como o uso em comum de cartões de crédito ou débito entre o casal, são ações que determinam maiores ou menores níveis de compartilhamento dos recursos.

Dentre as limitações deste estudo, as características da amostra não são representativas da população nacional, tanto em relação à distribuição geográfica e escolaridade, quanto ao nível socioeconômico e à situação conjugal. A população que participou deste estudo foi majoritariamente residente do Sul do Brasil, com alto poder aquisitivo, em uma relação oficializada a nível civil e/ou religioso, com ensino superior e/ou pós-graduação. Essas características podem ter sido um viés de seleção para o engajamento na pesquisa, conforme o esclarecimento e interesse em relação ao tema. Em segundo lugar, o que caracteriza a inovação deste estudo também constitui uma limitação, pois a indisponibilidade de instrumentos que avaliem os construtos aqui trabalhados não permite que seja verificada a validade concorrente das dimensões em sua totalidade.

Ademais, verificou-se que a EMDC apresentou evidências de validade de construto e precisão para avaliar os estilos de manejo de dinheiro individual ou compartilhado na conjugalidade. Os resultados expressos justificam a sua utilização para investigar a forma como os casais lidam com os recursos financeiros, significando uma importante contribuição para o estudo das relações conjugais no contexto nacional. Sugere-se futuras pesquisas, visando a padronização da escala por meio de amostra representativa da população brasileira.

\section{Referências}

Addo, F. R., \& Sassler, S. (2010). Financial arrangements and relationship quality in lowincome couples. Family Relations, 59(4), 408-423. doi:10.1111/j.1741-3729.2010.00612.x

Associação Brasileira das Entidades dos Mercados Financeiros e de Capitais, Anbima (2018). O raio-x do investidos brasileiro. Recuperado de https:// www.anbima.com.br/pt_br/especial/raio-x-do-investidor-2018.htm

Archuleta, K. L. (2013). Couples, money, and expectations: Negotiating financial management roles to increase relationship satisfaction. Marriage and Family Review, 49(5), 391-411. doi:10.1080/01494929.2 013.766296

Archuleta, K. L. (2008). The impact of dyadic processes and financial management roles on farm couples. (Tese de doutorado) Kansas State University, Manhattan, Kansas, Estados Unidos da América. doi:10.1080 /01494929.2013.766296

Barros, S. C., Borges, L. O., \& Estramiana, J. L. A. (2017). Níveis de análise nos estudos sobre os significados do dinheiro. Athenea Digital, 17(3), 131-148. doi:10.5565/rev/athenea.1858

Batista-Foguet, J. M., Coenders, G., \& Alonso J. (2004). Análisis fatorial confirmatória: Su utilidad em la validación de cuestionarios relacionados a la salud. MedClin(Barc), 122, 21-27.

Bereza, E., Martins, J. P., Moresco, L., \& Zanoni, S. H. M. S. (2005) A influência da comunicação no relacionamento conjugal. Arquivos de Ciências da Saúde da UNIPAR, 9(1), 31-0. doi: 10.25110/arqsaude. v911.2005.216

Brasil, Presidência da República (2019). Decreto $\mathbf{n}^{\mathbf{o}}$ 9.661, de $1^{\circ}$ de janeiro de 2019. Recuperado de http://www.planalto.gov.br/ccivil_03/_Ato20192022/2019/Decreto/D9661.htm

Brasil, Conselho Nacional de Saúde. (2016). Resolução no 510 de 07 de abril de 2016, Brasilia, 2016. 
Recuperado de http://conselho.saude.gov.br/resolucoes/2016/Reso510.pdf

Brasil, Conselho Nacional de Saúde. (2012) Resolução no 466 de 12 de dezembro de 2012, Brasília, 2012. Recuperado de http://conselho.saude.gov.br/resolucoes/2012/reso466.pdf

Brown, T. A. (2006). Confirmatory Factor Analysis for Applied Research, New York: The Guilford Press.

Burgoyne, C. B., Clarke, V., Reibstein, J., \& Edmunds, A. (2006). "All my wordly goods I share with you: Managing Money at the transition to heterossexual marriage. The Sociological Review, 54(4), 619-637. doi: 10.1111/j.1467-954X.2006.00663.x

Byrne, B. M. (2010). Structural equation modeling with AMOS: Basic concepts, applications, and programming. Nova Iorque: Routledge, Taylor \& Francis

Cardoso, L. B. S. A, \& Bucher-Maluschke, J. S. N. F. (2017). O casal face as finanças: Revisão da literatura. Rev. Nufen: Phenom. Interd, 9(3), 177-187 doi: 10.26823/RevistadoNUFEN.vol09.n03revir22

Cenci, C. M. B, \& Habigzang, L. F. (2019). Dinheiro e conjugalidade: Posicionamentos e estratégias conjugais para resolução de dilemas financeiros. Gerais: Revista Interinstitucional de Psicologia, 12(1), 159-174. Recuperado de http:// pepsic.bvsalud.org/scielo.php?script $=$ sci_ abstract\&pid $=$ S1983-82202019000100012\&lng= $\mathrm{pt} \& \mathrm{nrm}=$ iso\&tlng $=\mathrm{pt}$

Cenci, C. M. B., Bona, C. S., Crestani, P., \& Habigzang, L. F. (2017). Dinheiro e conjugalidade: Uma revisão sistemática da literatura. Temas Em Psicologia, 25(1), 385-399. doi:10.9788/TP2017.1-20

Cenci, C. M. B, Pauli, J., \& Folle, P. D. (2018). Conjugalidade negociada: Elementos para compreensão do significado que casais atribuem ao dinheiro. Actualidades en Psicología, 32(124), 76-91. doi:10.15517/ ap.v32i124.28392

Confederação Nacional do Comércio de Bens, Serviços e Turismo, CNC (2019). Pesquisa de Endividamento e Inadimplência do Consumidor - PEIC. Recuperado de http://www.cnc.org.br/editorias/ economia/pesquisas/pesquisa-de-endividamento-e-inadimplencia-do-consumidor-peic-maio-de

Crespo, C., Narciso, I., Ribeiro, M., \& Costa, M. (2006). Desenvolvimento da escala de dimensões da intimidade: Primeiro estudo empírico. Psychologica,
41, 45-63. Recuperado de https://hdl.handle. net/1021696730

Comissão de Valores Mobiliários, CVM (2018). Cenário da poupança e dos investimentos brasileiros. Recuperado de http://www.cvm.gov.br/ export/sites/cvm/menu/investidor/estudos/ pesquisas/20181002_estudo_spc_cenario_da_ poupanca_e_dos_investimentos_dos_brasileiros. pdf

Diniz, G. (2011) Conjugalidade e violência: Reflexões sobre uma ótica de gênero. Em T. Féres-Carneiro (Eds.). Casal e Familia: Conjugalidade, parentalidade e psicoterapia (pp 11-26). Porto Alegre, RS: Casa do Psicólogo.

Fères-Carneiro, T; \& Diniz Neto, O. (2010). Construção e dissolução da conjugalidade: Padrões relacionais. Paidéia (Ribeirão Preto), 20(46), 269-278. doi: 10.1590/S0103-863X2010000200014

Fères-Carneiro, T. (1998). Casamento contemporâneo: O difícil convívio da individualidade com a conjugalidade. Psicologia: Reflexão e Crítica, 11(2), 379-394. doi:10.1590/S0102-79721998000200014

Garbin, A. D. S., Mara, C., Cenci, B., \& Luz, S. K. (2015). Dinheiro e conjugalidade, 7(1), 72-78. Recuperado de http://www.bibliotekevirtual.org/index.php/201302-07-03-02-35/2013-02-07-03-03-11/1187-psicoimed/v07n01/12200-dinheiro-e-conjugalidade.htm

Haack, K. R., \& Falcke, D. (2013). Infidelid@ ade.com: Infidelidade em relacionamentos amorosos mediados e não mediados pela internet. Psicologia em Revista, 19(2), 305-327. doi: 10.5752/P.1678-9563.2013v19n2p305

Hair, J. F., Black, W. C., Babin, B. J., Anderson, R. E., \& Tatham, R. L. (2009). Análise multivariada de dados. Porto Alegre, RS: Bookman Editora

Harth, J., Mosmann, C. P., \& Falcke, D. (2016). Manejo do dinheiro pelo casal e infidelidade financeira. Estudos e Pesquisas em Psicologia, 16(1), 260-276. Recuperado de http://pepsic.bvsalud. org $/$ scielo.php?script $=$ sci_arttext\&pid $=\mathrm{S} 1808$ 42812016000100015

Harth, J., \& Falcke, D. (2017). Manejo do dinheiro e qualidade conjugal. Interação em Psicologia, 21(1), 9-18. doi: 10.5380/psi.v21i1.32215

Hollist, C. S., Falceto, O. G., Ferreira, L. M., Miller, R. B., Springer, P. R., Fernandes, C. L., \& Nunes, N. 
A. (2012). Portuguese translation and validation of the Revised Dyadic Adjustment Scale. Journal of Marital and Family Therapy, 38(s1), 348-358. doi: 10.1111/j.1752-0606.2012.00296.x

Instituto Brasileiro de Geografia e Estatística [IBGE]. (2015.). Pesquisa Nacional de Amostra por Domicílios. Recuperado de https://ww2.ibge.gov.br/ home/estatistica/populacao/trabalhoerendimento/pnad2015/default.shtm

Kline, R. B. (2005). Principles and practice of structural equation modeling ( $2 \mathrm{a}$ ed.). New York: The Guilford Press

Levine, S. B. (1992). Sexual life: A clinician guide. New York: Plenum Press

Luz, S. K.., \& e Mosmann, C. P. (2018) Funcionalidade e comunicação conjugal em diferentes etapas do ciclo de vida. Revista da SPAGESP, 19(1), 21-34. Recuperado de http://pepsic.bvsalud.org/scielo.php?script=sci_ abstract\&pid $=$ S1677-29702018000100003\&lng $=$ pt \&nrm=iso

Moss, B. F., \& Schwebel, A. I. (1993). Defining intimacy in romantic relationships. Family Relations: An Interdisciplinary Journal of Applied Family Studies, 42(1), 31-37. doi: $10.2307 / 584918$

Olson, D. H. (2000). Circumplex model of marital and family systems. Journal of Family Therapy, 22(4), 144167 doi:10.1111/1467-6427.00144

Pahl, J. (1989), Money and Marriage. London: Macmillan.

Pahl, J. (2000). Couples and their money: Patterns of accounting and accountability in the domestic economy. Accounting, Auditing \& Accountability Journal, 13(4), 502-517. doi: 10.1108/09513570010338078

Pahl, J. (2005). Individualisation in couple finances: Who pays for the children? Social Policy and Society, 4(04), 381. doi:10.1017/S1474746405002575

Papp, L. M., Cummings, E. M., \& Goeke-Morey, M. C. (2009). For richer, for poorer: Money as a topic of marital conflict in the home. Family Relations, 58(1), 91-103. doi:10.1111/j.1741-3729.2008.00537.x

Pasquali L. (2011) Psicometria: Teoria dos testes na psicologia e na educação. Petrópolis: Vozes.

Pereira-Silva, N. L. (2015). Estresse e ajustamento conjugal de casais com filho (a) com Síndrome de Down. Interação em Psicologia, 19(2), 297-308. doi: 10.5380/ psi.v19i2.32747
Peruchi, R. C., Donelli, T. M. S., \& Marin, A. H. (2016). Ajustamento conjugal, relação mãe-bebê e sintomas psicofuncionais no primeiro ano de vida. Quaderns de Psicología, 18(3), 55-67. doi:10.5565/rev/ qpsicologia.1363

Prager, K. J. (2014). The dilemas of intimacy: Conceptualization, assessment and treatment. New York and London: Routledge.

Raykov, T., \& Marcoulides, G. A. (2000). A first course in structural equation modeling. Mahwah, NJ, US: Lawrence Erlbaum Associates Publishers.

Razera, J., Cenci, C. M., \& Falcke, D. (2015). Manejo de dinheiro: Possíveis relações com o ajustamento e a violência em casais. Perspectivas em Psicologia, 19(2), 3-17 Recuperado de http://www.seer.ufu. $\mathrm{br} /$ index.php/perspectivasempsicologia/article/ view/32492

Reppold, C. T., Gomes, C. M. A., Seabra, A. G., Muniz, M., Valentini, F., \& Laros, J. A. (2015). Contribuições da psicometria para os estudos em neuropsicologia cognitiva. Psicologia: teoria e prática, 17(2), 94-106.

Rosa, I. R., \& Milani, B. (2014). Significado do dinheiro: Um estudo sobre o comportamento de estudantes de nível superior. Revista de Administração IMED, 4(3), 369-380. doi: 10.18256/2237-7956/raimed. v4n3p369-380

Rosado, J. S., \& Wagner, A. (2015). Qualidade, ajustamento e satisfação conjugal: Revisão sistemática da literatura. Pensando Famílias, 19(2), 21-33. Recuperado de http://pepsic.bvsalud.org/scielo. php?script $=$ sci_arttext\&pid=S1679-494X2015000 200003\&lng $=$ pt\&tlng $=$ pt

Sattler, M. K., Tavares, A. C. C. N., \& Silva, I. M. da. (2017). A infidelidade no relacionamento amoroso: Possibilidades no trabalho clínico com casais. Pensando Famílias, 21(1), 162-175 Recuperado de http:// pepsic.bvsalud.org/scielo.php?script $=$ sci_ arttext\&pid=S1679-494X2017000100013\&lng=pt $\& \operatorname{lng}=\mathrm{pt}$.

Serviço de Proteção ao Crédito Brasil [SPC], \& Comissão Nacional dos Dirigentes Lojistas [CDL]. (2017). Indicador de Reserva Financeira. Recuperado de https://wnw.spcbrasil.org.br/.../mp.../ Indicador-de-Reserva-Financeira-mar_18v7.pdf

Psico-USF, Bragança Paulista, v. 26, n. 2, p. 305-318, abr./jun. 2021 
Serviço de Proteção ao Crédito Brasil [SPC], \& Comissão Nacional dos Dirigentes Lojistas [CDL] (2018). Indicador de Reserva Financeira. Recuperado de https://www.spcbrasil.org.br/wpimprensa/ wp-content/uploads/2018/08/An\%C3\%A1lise_-Indicador-de-Reserva-Financeira-jul_18.pdf

Torman, V., Coster, R., \& Riboldi, J. (2012). Normalidade de variáveis: Métodos de verificação e comparação de alguns testes não paramétricos por simulação. Clinical \& Biomedical Research, 32(2). Recuperado de https://seer.ufrgs.br/hcpa/article/ view/29874

Troost, A., Vermulst, A. A., Gerris, J. R. M., \& Matthijs, K. (2005). The Dutch Marital Satisfaction and Communication Questionnaire: A Validation Study. Psychological Belgica, 45(3), 185-206. doi:10.5334/ pb-45-3-185
Vogler, C., \& Pahl, Jan. (1993). Social and economic change and the organisation of money within marriage. Work Employment and Society, 7(1), 71-95. doi: 10.1177/0950017093007001005

Vogler, C., Lyonette, C., \& Wiggins, R. D. (2008). Money, power and spending decisions inintimate relantionships. The Sociological Review, 56(1), doi:10.1111/j.1467-954X.2008.00779.x

Yodanis, C., \& Lauer, S. (2007). Economic inequality in and outside of marriage: Individual resources and institutional context. European Sociological Review, 23(5), 573-583. doi: 10.1093/esr/jcm021

Recebido em: 22/07/2019

Reformulado em: 01/05/2020

Aprovado em: 15/06/2020 
Sobre as autoras:

Lídia Käfer Schünke é Psicóloga, Mestre e Doutoranda em Psicologia Clínica pela Universidade do Vale do Rio dos Sinos, São Leopoldo, Bolsista da Coordenação de Aperfeiçoamento de Pessoal de Nível Superior (CAPES); Pesquisadora do Núcleo de Estudos em Casais e Familias (NECAF) Universidade do Vale do Rio dos Sinos, São Leopoldo. ORCID: https://orcid.org/0000-0002-3770-5982

E-mail: lidia.kafer@gmail.com

Rebeca Veras de Andrade Vieira é Psicóloga, Mestre e Doutora em Psicologia pelo Programa de Pós-Graduação em Psicologia da Universidade Federal do Rio Grande do Sul (UFRGS); Programa de Doutorado Sanduíche (CAPES) na Johns Hopkins School of Medicine (USA); Formação em Psicologia Hospitalar (Hospital de Clínicas de Porto Alegre) Especialista em Psicoterapia Cognitivo-Comportamental pelo Instituto WP; Bolsista pelo Programa Nacional de Pós-Doutorado CAPES na Universidade do Vale do Rio dos Sinos (UNISINOS).

ORCID: https://orcid.org/0000-0003-2907-8699

E-mail: rebecavieirapsico@gmail.com

Clarisse Pereira Mosmann é Psicóloga, Doutora em Psicologia pela Pontifícia Universidade Católica do Rio Grande do Sul (PUCRS), Pós-doutorado na Universidade de Girona e na UFRGS, especialista em Terapia de Casal e Família pela Escuela de Formación en Terapia Familiar - STIRPE. Coordenadora Executiva do Programa de Pós-Graduação e Professora do Curso de Psicologia da Universidade do Vale do Rio dos Sinos (UNISINOS). Coordenadora do Núcleo de Estudos em Casais e Famílias (NECAF)Universidade do Vale do Rio dos Sinos, São Leopoldo.

ORCID: https://orcid.org/0000-0002-9275-1105

E-mail: clarissepm@unisinos.br

Contato com as autoras:

Programa de Pós Graduação em Psicologia Unisinos

Av. Unisinos, 950 - Sala e01 215, Cristo Rei

São Leopoldo-RS, Brasil

CEP: 93022-750 